\title{
Which risk engines are best to assess CVD risk in diabetes?
}

\author{
Parinya Chamnan, Rebecca K. Simmons and Simon J. Griffin
}

The News and Views article by Ray and Sattar (Diabetes: Which risk engines should be used to assess patients with diabetes? Nat. Rev. Endocrinol. 5, 302-303; 2009) ${ }^{1}$ raises a number of interesting points regarding the role of cardiovascular disease (CVD) risk assessment tools in the prevention of CVD among people with type 2 diabetes mellitus (T2DM). The authors suggest that the UK Prospective Diabetes Study (UKPDS) risk engine has several advantages over the Framingham risk score (FRS), particularly as it incorporates a measure of glycemia and duration of diabetes mellitus, alongside other traditional risk factors. However, our systematic review demonstrates that scant evidence exists to suggest that diabetesspecific risk tools estimate CVD risk more accurately than those developed in the general population. ${ }^{2}$ Validation studies comparing the predictive performance of the FRS and the UKPDS risk engine report conflicting results. ${ }^{3-5}$ Furthermore, adding a measure of glycemia to the FRS improves discrimination slightly in men but not in women, without significant improvement in reclassification of risk category. ${ }^{6}$

The authors' conclusion that $2 \%$ of patients with T2DM might be below a treatment threshold, and hence denied medication (depending on choice of risk scores) is an oversimplification of the issue. While the total proportions with a modeled 10 -year CVD risk $\geq 20 \%$ may be similar, two different scores may identify completely different individuals, thus statistics such as the Net Reclassification Improvement are more informative.

Ray and Sattar advocate measurement of lifetime CVD risk in order to circumvent problems related to changes in CVD risk over time. Modeling lifetime risk is prone to many sources of uncertainty. Changes in the distribution of CVD risk factors and treatments over time, both within and between populations, could limit the predictive accuracy. Furthermore, it might be more clinically relevant and also more persuasive for patients to know CVD risk and treatment benefits over a shorter period.

Ray and Sattar conclude by suggesting that future studies on T2DM should incorporate data on emerging prognostic biomarkers to help improve CVD risk prediction and thus risk reduction. However, very few novel biomarkers improve risk prediction models over and above the inclusion of traditional risk factors, and any improvement is likely to be marginal.,8 There may be little to gain from developing novel CVD risk scores, incorporating expensive biomarkers, in historical cohorts including individuals dissimilar to patients in whom the risk score would be used. ${ }^{9}$ More precise risk prediction by itself will not improve health outcomes. Indeed, there is no strong evidence that a CVD risk assessment performed by a clinician improves CVD-related health outcomes. ${ }^{10}$ Further research might address other potential uses of risk scores, for example, to better understand the interaction between clinicians and patients when CVD risk has been assessed, and the effects on physician prescribing and patient health behaviors such as diet, smoking, physical activity and medication adherence.

In summary, diabetes-specific risk scores do not predict CVD in people with T2DM more accurately than risk scores developed in the general population. Future research might focus on exploring patient-provider perceptions and responses to information about CVD risk.
MRC Epidemiology Unit, Institute of Metabolic Science, Box 285, Addenbrooke's Hospital, Cambridge CB2 OQQ, UK (P. Chamnan, R. K. Simmons, S. J. Griffin)

Correspondence to: P. Chamnan parinya.chamnan@mrc-epid.cam.ac.uk

doi:10.1038/nrendo.2009.100-c1

Competing interests

The authors declare no competing interests.

1. Ray K. K. \& Sattar, N. Diabetes: Which risk engines should be used to assess patients with diabetes? Nat. Rev. Endocrinol. 5, 302-303 (2009).

2. Chamnan, P., Simmons, R. K., Sharp, S. J., Griffin S. J. \& Wareham, N. J. Cardiovascular risk assessment scores for people with diabetes: a systematic review. Diabetologia 52, 2001-2014 (2009).

3. Guzder, R. N., Gatling, W., Mullee, M. A., Mehta R. L. \& Byrne, C. D. Prognostic value of the Framingham cardiovascular risk equation and the UKPDS risk engine for coronary heart disease in newly diagnosed Type 2 diabetes: results from a United Kingdom study. Diabet. Med. 22, 554-562 (2005).

4. Simmons, R. K. et al. Performance of the UK Prospective Diabetes Study Risk Engine and the Framingham Risk Equations in Estimating Cardiovascular Disease in the EPIC-Norfolk Cohort. Diabetes Care. 32, 708-713 (2009).

5. Stephens, J. W. et al. Cardiovascular risk and diabetes. Are the methods of risk prediction satisfactory? Eur. J. Cardiovasc. Prev. Rehabil. 11, 521-528 (2004).

6. Simmons, R. K. et al. Evaluation of the Framingham risk score in the European Prospective Investigation of Cancer-Norfolk cohort: does adding glycated hemoglobin improve the prediction of coronary heart disease events? Arch. Intern. Med. 168, 1209-1216 (2008).

7. Folsom, A. R. et al. An assessment of incremental coronary risk prediction using C-reactive protein and other novel risk markers: the atherosclerosis risk in communities study. Arch. Intern. Med. 166, 1368-1373 (2006).

8. Shah, T. et al. Critical appraisal of CRP measurement for the prediction of coronary heart disease events: new data and systematic review of 31 prospective cohorts. Int. J. Epidemiol. 38, 217-231 (2009).

9. Lloyd-Jones D. M. \& Tian, L. Predicting cardiovascular risk: so what do we do now? Arch. Intern. Med. 166, 1342-1344 (2006).

10. Brindle, P., Beswick, A., Fahey, T. \& Ebrahim, S. Accuracy and impact of risk assessment in the primary prevention of cardiovascular disease: a systematic review. Heart 92, 1752-1759 (2006). 